\title{
Bidirectional DC-DC Converter with Digital Droop Parameterization
}

\author{
Michael Jupke, Andrea Reindl, Hans Meier, Michael Niemetz \\ Faculty of Electrical Engineering and Information Technology \\ Ostbayerische Technische Hochschule Regensburg, Germany \\ michael1.jupke@st.oth.regensburg.de, andreareindl@ieee.org, \{hans.meier, michael.niemetz\}@oth-regensburg.de
}

\begin{abstract}
The key for decentralized battery systems is a robust and communication-less control strategy for autonomous power sharing of parallel-connected DC-DC converters. Battery systems improve the reliability and quality of power supply in renewable energy systems and enable power supply for off-grid, mobile applications, including islanded grids, home storage, and electric vehicles. In many cases, components with different electrical properties require different voltage levels. An adaptation is consequently essential and is normally implemented in DC grids for the batteries via bidirectional DC-DC converters. The power flow in both directions can thus be ensured. To achieve a power distribution in parallel connected DC-DC converters, a droop control in the form of a virtual internal resistor can be used. This paper presents a novel approach of a DCDC converter with a digitally parameterizable droop resistor, whose voltage regulation is based on an analog operational amplifier circuit to ensure low delays and robustness. The droop resistor is adjusted with a microcontroller, which offers the possibility to apply a higher-level control for load sharing via an interface. Mathematical correlations are used to clearly define the parameters of the control. Furthermore, the circuit was completely simulated and tested in the hardware setup. The shown results verify the functionality and indicate only minor deviations. Therefore, this circuit is important for future use in distributed battery systems.
\end{abstract}

Keywords-DC-DC power converters, Power stage, Bidirectional power flow, Automatic voltage control, Droop control, Load sharing, Microgrids, Battery management systems, Microcontrollers

\section{INTRODUCTION}

Using renewable energy sources is a promising solution to reduce greenhouse gas emissions. In this context, batteries are essential to improve the quality and reliability of the power supply in energy systems. In many applications, including residential battery storage systems, uninterruptible power supply, island grids, and electric vehicles, the battery system maintains the energy balance.

In order to interconnect energy generators, electrical loads, and batteries with different electrical characteristics, power electronic converters are required between each component and the common DC line. Bidirectional DC-DC converters provide the power flow in the charging and discharging direction $[1-3]$. To optimize reliability and extend battery life, effective power sharing between the batteries depending on the current state, e.g., state of charge or state of health, is required $[4,5]$.

The control strategy is essential for the maintenance of the bus voltage, the power distribution between the generators, batteries and loads, and the charge balance between the batteries. With regard to the reliability and robustness of the battery system, communication-less, decentralized control strategies offer particular advantages $[6,7]$.
Droop control is an established method for load sharing of parallel connected components and voltage control [8, 9]. It is based on local measurements and does not require communication between the components [10]. The used droop characteristic divides the load and determines the power of the DC-DC converters. The droop characteristic corresponds to a virtual internal resistance and enables the determination of the required power at a locally acquired reference voltage (Fig. 1). The operating point results from the reference voltage and the droop resistance characteristic $\left(R_{\mathrm{D} 1}\right.$ and $\left.R_{\mathrm{D} 2}\right)$ to set the output voltage (VOUT) of the DC-DC converter.

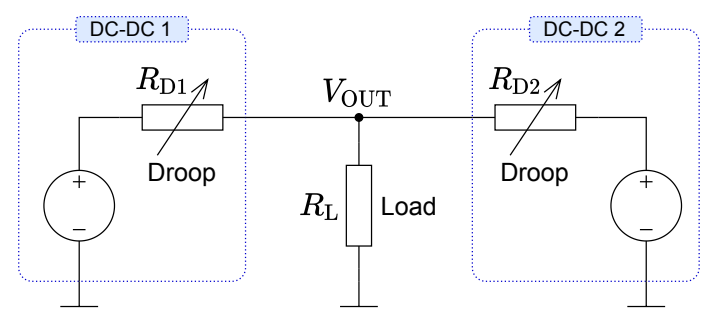

Fig. 1. Equivalent circuit diagram for two converters using droop control, adapted from [19].

By varying the slope of the droop characteristic it is possible to load the DC-DC converters differently. To ensure safe battery operation and effective battery life, it is necessary to adjust the droop characteristic in battery systems depending on the corresponding battery condition [11]. In this paper, the variance of the droop slope is realized by a digitally adjustable potentiometer via the microcontroller $(\mu \mathrm{C})$. This allows communication-less load sharing depending on the battery state and the combination of different battery technologies.

Concerning the reliability and fail-safety of the battery system, analog voltage regulation is targeted to ensure stable operation even in case of a communication or a microcontroller failure. Therefore, an efficient bidirectional DC-DC converter is proposed, which realizes an analog voltage regulation with a digitally parametrizable droop resistor. In addition, the digitally controlled DC-DC converter enables distributed control strategies in which one computing node manages the load of the individual energy storage devices and monitors the grid voltage [12].

In Section II, the bidirectional DC-DC converter is described and in Section III, the system architecture is presented. In Section IV, the control methods are discussed and in Section V, experiments and results in simulation and hardware are shown. Afterward, the results are discussed in Section VI. 


\section{DesCRIPTION OF THE BidiRECTIONAL DC-DC CONVERTER}

Basically, there are two groups of DC-DC converters: isolated and non-isolated. Furthermore, a classification between single-phase and multi-phase converters can be made. A multiphase DC-DC converter is represented by an array of individual converters which are controlled by a phaseshifted signal. This results in high efficiency with a wide power range at the same time. In addition, splitting into multiple power paths reduces copper losses, current ripple, and electromagnetic emissions [13]. The most common circuit topology for the power stage of non-isolated bidirectional DCDC converters is the half-bridge. The reasons for this are the high efficiency due to lower switching and ohmic losses in the inductance and other active components. [3,14,15]

The employed chip LM5170 [16] is a current controller for non-isolated bidirectional DC-DC converters with half-bridge topology. With its two channels (phases), it directly drives two power stages. Fig. 2 illustrates the operation of a single channel.



Fig. 2. Functional representation of the wiring for one channel of the bidirectional power stage with the LM5170.

The circuit represents a combination of a buck and a boost converter. In buck mode, $T_{1}$ acts as a switch with the required duty cycle and $T_{2}$ is continuously off. In boost mode, $T_{2}$ is triggered while $T_{1}$ is always off. Thereby, the magnetic field of the inductor $L$ acts as energy storage. The circuit already provides a current control with the shunt resistor $R_{\text {SHUNT }}$ as the measuring element. The external voltage signal $V_{\text {ISETA }}$ sets the target current at the LV-port while the digital signal $D I R$ defines the current direction at the LV-port and thus the direction of the power flow (Fig. 2). [16] Other signals are only relevant for further control requirements and are not considered here.

To verify the intended functionality quickly, an evaluation board [17] is used as a bidirectional DC-DC converter. This offers the advantage that essential pins of the LM5170 can be easy connected via a pin header. The printed circuit board DC-DC converter operates with a maximum current of $60 \mathrm{~A}$ at the LV-port. The voltage range at the HV-port is between $6 \mathrm{~V}$ and $75 \mathrm{~V}$, and at the LV-port between $3 \mathrm{~V}$ and $48 \mathrm{~V}$. The evaluation board already provides an optional voltage control, but this is designed for fixed values of $14.5 \mathrm{~V}$ at the $\mathrm{LV}$-port and $50.5 \mathrm{~V}$ at the $\mathrm{HV}$-port. The lead-out pin $V_{\text {ISETA }}$ is preconfigured in such a way that a current of $I_{\mathrm{LV}}= \pm 40 \mathrm{~A}$ flows at the LV-port when a signal of $V_{\text {ISETA }}=1 \mathrm{~V}$ is applied. The current is positive in buck mode and negative in boost mode. [17]

\section{System ARChitecture}

Fig. 3 shows an application of a bidirectional DC-DC converter with adjustable droop resistance within a Battery Management System (BMS) consisting of several nodes, which act as power sources or power sinks. A CAN bus is used for communication between the individual nodes. In a node, a battery is connected to the DC line via the converter. The evaluation board described above is used as the bidirectional DC-DC converter component in this setup. To manage the converter a microcontroller based control board equipped with all components required for voltage control and CAN communication was designed.

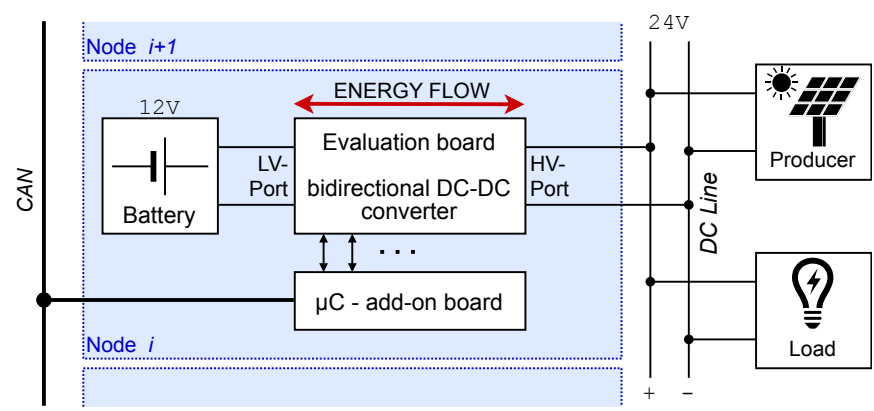

Fig. 3. Microcontroller based DC-DC evaluation board integrated as a node of a BMS with battery, producer, load, and CAN bus.

With this architecture, many different energy sources and sinks can be connected to the common DC line. A BMS can thereby take over the higher-level management of the individual nodes. Specifically, each node receives separate instructions on how to charge or discharge its battery. The parameter settings depend on the output voltage and the value of the desired internal resistance (droop resistance $R_{\mathrm{D}}$ ).

For improved flexibility in future applications, the voltage control based on adjustable droop resistance was realized for both, the LV-port and the HV-port. However, the circuit design for the $\mathrm{HV}$-port is analogous besides adapted component values. Therefore in the following, we focus on the LV-port design labeling it as $V_{\text {OUT }}$ and the current $I_{\mathrm{LV}}$ as $I_{\mathrm{OUT}}$.

\section{PRINCIPLE OF THE CONTROL}

Fig. 4 shows the basic control structure of a node for the application of a BMS, as described in section III. The control can be divided into an analog and a higher-level digital control. The microcontroller [18] communicates with other nodes via the CAN network and sets the parameters for the power distribution accordingly. For this purpose, the microcontroller specifies the analog reference voltage $V_{\mathrm{REF}}$ via a DAC. It also defines the set point for the droop resistance with the $\mathrm{I}^{2} \mathrm{C}$ signal for $R_{\text {POT }}$. The analog voltage control with adjustable droop resistance generates the output variable $V_{\text {ISETA }}$ from the reference voltage $V_{\mathrm{REF}}$, the current output voltage $V_{\mathrm{OUT}}$ and the specific set point $R_{\text {POT }}$ of the droop resistance. The DC-DC converter shows in buck mode a steady-state gain conductance factor $F_{\mathrm{DCDC}}$ of:

$$
F_{\mathrm{DCDC}}=\frac{I_{\mathrm{LV}}}{V_{\mathrm{ISETA}}}=40 \mathrm{~A} / \mathrm{V}
$$

Due to the placement of the current sense resistor $R_{\text {SHUNT }}$ of the DC-DC converter (cf. Fig. 2), in equation 1 the losses of the DC-DC converter must also be taken into account when 




Fig. 4. Digital and analog control loop structure placed on the $\mu \mathrm{C}$ - add-on board to steer the DC-DC converter (node) with a connected load.

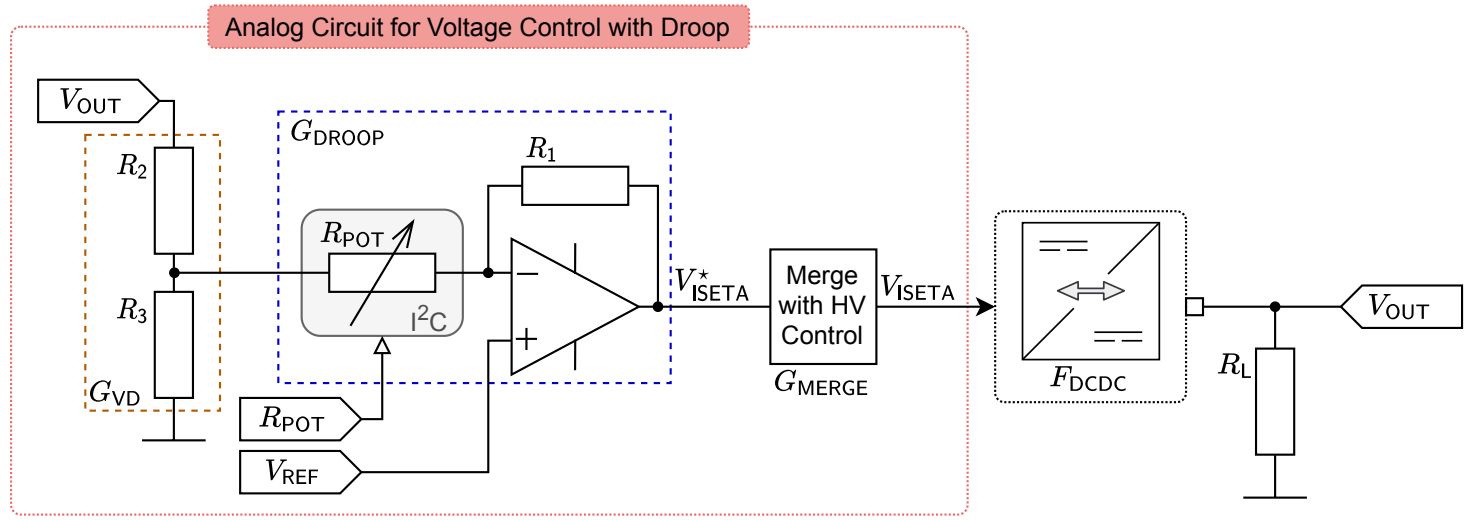

Fig. 5. Analog circuit for voltage control with droop in detailed view with the labeling of the corresponding gain factors.

operating in boost mode. For better comprehensibility, only the buck mode is considered from here on.

The internal current controller of the bidirectional DC-DC converter generates the output current $I_{\mathrm{OUT}}$ from the control variable $V_{\text {ISETA }}$ based on equation 1 . In combination with the load, in this case the impedance of the DC line, the output voltage $V_{\text {OUT }}$ results.

Furthermore, the microcontroller monitors the output voltage $V_{\text {OUT }}$ and the output current $I_{\text {OUT }}$. This allows to communicate the values among the nodes and offer the possibility for a higher-level digital control.

\section{A. Analog Voltage Control with Droop}

In Fig. 5 the hardware structure of the analog control for the LV-port is shown. Besides regulating the voltage, the main requirement is to generate a voltage drop depending on the output current. This behavior is achieved by limiting the DC gain in the voltage control loop [19].

In general, when using a proportional controller there is always a permanent control error, which generates a currentdependent voltage loss. This behavior can be explained by the following simplified example: $x$ describes the control result (output voltage) of a proportional controller with amplification $K_{\mathrm{PR}}$ at an existing system with amplification $K_{\mathrm{PS}} . w$ denotes the given setpoint of the output voltage. In addition, the system $K_{\mathrm{PS}}$ consists of the DC-DC converter as the current source and the load resistor $R_{\mathrm{L}}$. Thus, the system $K_{\mathrm{PS}}$ is loaddependent and hence the desired output voltage $x$ is a function of the load $R_{\mathrm{L}}$. Equation 2 specifies the output variable $x$ under the influence of the residual control deviation.

$$
x=\frac{w \cdot K_{\mathrm{PR}} \cdot K_{\mathrm{PS}}}{1+K_{\mathrm{PR}} \cdot K_{\mathrm{PS}}}
$$

Moreover, the resulting internal resistance can be calculated with equation 3 .

$$
R_{\mathrm{D}}=\frac{(w-x) \cdot R_{\mathrm{L}}}{x}
$$

By substituting equation 2 into equation 3 , it can be shown that the internal resistance $R_{\mathrm{D}}$ is independent of the load $R_{\mathrm{L}}$ and the given output voltage $w$. As a result, this behavior is identical to a voltage source with internal resistance. Consequently, the equivalent internal resistance is the droop resistance $R_{\mathrm{D}}$ of the DC-DC converter.

In the concrete realization, cf. Fig. 5, the voltage of the output $V_{\text {OUT }}$ is first scaled down via a voltage divider with the amplification factor $G_{\mathrm{VD}}$. In combination with the reference voltage $V_{\mathrm{REF}}$, the control derivation is created, which is amplified accordingly via the circuitry of the operational amplifier and generates the intermediate value $V_{\text {ISETA }}^{\star}$. It must be noted that the voltage divider is directly loaded with the operational amplifier circuit. Thus, the value of the resistor $R_{\mathrm{POT}}$ has a linear influence on $G_{\mathrm{VD}}$. The circuit therefore represents a bandwidth-limited proportional controller. Since the components for frequency compensation do not influence the DC operating point of the circuit $[19,20]$, they are omitted in Fig. 5. To change the value of $R_{\mathrm{D}}$, only the gain factor of the proportional controller $G_{\mathrm{DROOP}}$ has to be adjusted. This is realized with a digital potentiometer $R_{\text {РОT }}$ [21], which is linked to the microcontroller via an $\mathrm{I}^{2} \mathrm{C}$ bus connection. The resistor values $R_{\text {POT }}$ are $\approx 35 \Omega$ (wiper resistance, specified 
in [21]) to $20 \mathrm{k} \Omega$ in 1024 steps. Thus, the gain factor is given by equation 4 :

$$
G_{\mathrm{DROOP}}=\frac{R_{1}}{R_{\mathrm{POT}}}
$$

As a separate voltage control is present for the buck and boost modes, the circuit contains an additional merging network to combine the output $V_{\text {ISETA }}^{\star}$ from the respective control parts to form the output $V_{\text {ISETA for the DC-DC }}$ converter. Due to a diode in the signal path for separating between the buck and boost control loop, the gain factor $G_{\text {MERGE }}$ of this network is not linear.

\section{B. Calculation of the Operating Point}

For the correct parameter set point determination, the mathematical relation between the droop resistance $R_{\mathrm{D}}$ and the output voltage $V_{\text {OUT }}$ is important. Since the gain factor of the voltage divider $G_{\mathrm{VD}}$ depends on the value of the potentiometer $R_{\mathrm{POT}}$, the following relationship applies:

$$
G_{\mathrm{VD}}=\left(1+R_{2} \cdot \frac{R_{3}+R_{\mathrm{POT}}}{R_{3} \cdot R_{\mathrm{POT}}}\right)^{-1}
$$

Using equation 2 and 3 , and substituting all gain factors of the circuit (cf. Fig. 5), the resulting droop resistance $R_{\mathrm{D}}$ can be described as follows:

$$
\begin{aligned}
R_{\mathrm{D}} & =\frac{1}{G_{\mathrm{VD}} \cdot G_{\mathrm{DROOP}} \cdot G_{\mathrm{MERGE}} \cdot F_{\mathrm{DCDC}}} \\
& =\frac{1}{G_{\mathrm{VD}}} \cdot \frac{R_{\mathrm{POT}}}{R_{1}} \cdot \frac{1}{G_{\mathrm{MERGE}}} \cdot \frac{1}{F_{\mathrm{DCDC}}}
\end{aligned}
$$

Equation 6 shows that $R_{\mathrm{D}}$ is independent of the reference voltage $V_{\mathrm{REF}}$ and can be varied with $R_{\mathrm{POT}}$. According to equation 7 , the following relationship applies to the output voltage $V_{\text {OUT }}$ :

$$
V_{\mathrm{OUT}}=\frac{V_{\mathrm{REF}}}{G_{\mathrm{VD}}} \cdot\left(1+\frac{1}{G_{\mathrm{DROOP}}}\right)-I_{\mathrm{OUT}} \cdot R_{\mathrm{D}}
$$

Finally, with equations 6 and 7, the parameters ( $V_{\text {OUT }}$ and $R_{\mathrm{D}}$ ) for the node are clearly defined and can be set properly via the microcontroller.

\section{EXPERIMENTS IN SimUlation AND HARDWARE}

To validate the entire circuit, a circuit simulation and measurements on a real test setup were performed. For both tests, an output characteristic field was recorded with different values of $R_{\text {POT }}$. The reference voltage $V_{\mathrm{REF}}$ was set to $1 \mathrm{~V}$. An output curve field was created in which the dependence of the output voltage $V_{\text {OUT }}$ on the output current $I_{\text {OUT }}$ is shown. It offers the advantage that the negative first derivative of the curve corresponds to the droop resistance $R_{\mathrm{D}}$.

\section{A. Simulation}

The circuit from Fig. 5 was simulated by the circuit simulation software LTSpice [22]. To generate an output characteristic field, the DC operating point was calculated for different load currents $I_{\text {OUT }}$ and different values of the potentiometer $R_{\text {POT }}$. In order to analyze the behavior of the remaining circuit parts, the voltage divider $\left(G_{\mathrm{VD}}\right)$ and the merging network $\left(G_{\text {MERGE }}\right)$ were also implemented with discrete components. Furthermore, a realistic model was used for the operational amplifier to keep the simulation error as low as possible. The DC-DC converter was modeled as a voltage-controlled current source with a gain factor of $F_{\mathrm{DCDC}}=40 \mathrm{~A} / \mathrm{V}$.

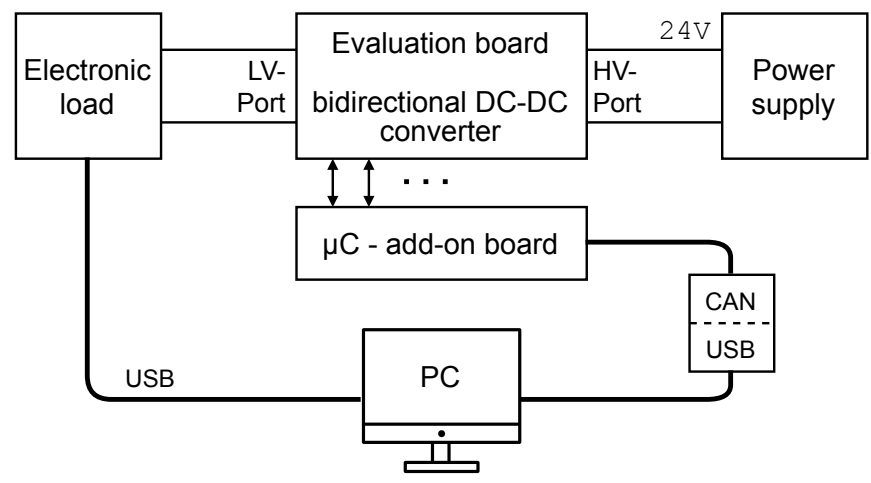

Fig. 6. Test setup of a node with the LM5170 evaluation board, the attached $\mu \mathrm{C}$ - add-on board, and a PC for processing the data.

\section{B. Realized Test Setup of one Node}

For the test setup shown in Fig. 6, the $\mu \mathrm{C}$ - add-on board was attached to the DC-DC converter [17] (Fig. 10). The $\mathrm{HV}$-port was supplied with a constant voltage of $24 \mathrm{~V}$. An electronic load [23], which can consume up to $400 \mathrm{~W}$, was connected to the LV-port and set to constant current operating mode. Using a test framework, the PC automatically applied the desired load current profiles and simultaneously recorded the output voltage $V_{\text {OUT }}$ and the output current $I_{\text {OUT }}$. The application of a 4-wire measurement setup ensured that the voltage loss on the lines did not falsify the results. Moreover, various resistance values of $R_{\mathrm{POT}}$ were configured by sending CAN messages to the node.

\section{Discussion of THE RESUlts}

First, the simulation results are presented, followed by the hardware results. Finally, a comparison is made.

\section{A. Simulation Results}

Fig. 7 shows the output characteristic field of the simulation for different resistance values of $R_{\text {POT }}$. The output voltage



Fig. 7. Simulation results from the LTspice simulation. Deviations from the linear behavior at low currents are caused by the offset voltage of the used amplifier. 




Fig. 8. Droop resistance values, obtained from the LTspice simulation.

$V_{\text {OUT }}$ for large values of $R_{\text {POT }}$ decreases more with increasing current $I_{\mathrm{OUT}}$ than for smaller values of $R_{\mathrm{POT}}$. Due to this behavior, load sharing can be achieved when multiple of these nodes are connected in parallel. Fig. 8 shows the negative numerical derivative of Fig. 7 and thus represents the droop resistor $R_{\mathrm{D}}$ depending on the output current. It is not constant through the output current range. There are two reasons for the shape of the curve: The sharp increase of the droop resistance $R_{\mathrm{D}}$ in the range $<2 \mathrm{~A}$ is caused by the offset voltage of the real operational amplifier. In the range $>2 \mathrm{~A}$, the offset voltage is no longer decisive for the imperfectly linear curve. In this range, the non-linear behavior of the diode in the merging network has a minor effect.

\section{B. Hardware Results}

Fig. 9 shows the determined output characteristic field from the hardware measurements. It shows that the array of curves is slightly shifted downwards and also to the left compared to Fig. 7. Such a shift occurs when the reference voltage $V_{\mathrm{REF}}$ is lower than $1 \mathrm{~V}$. This relationship is also proven by equation 7.

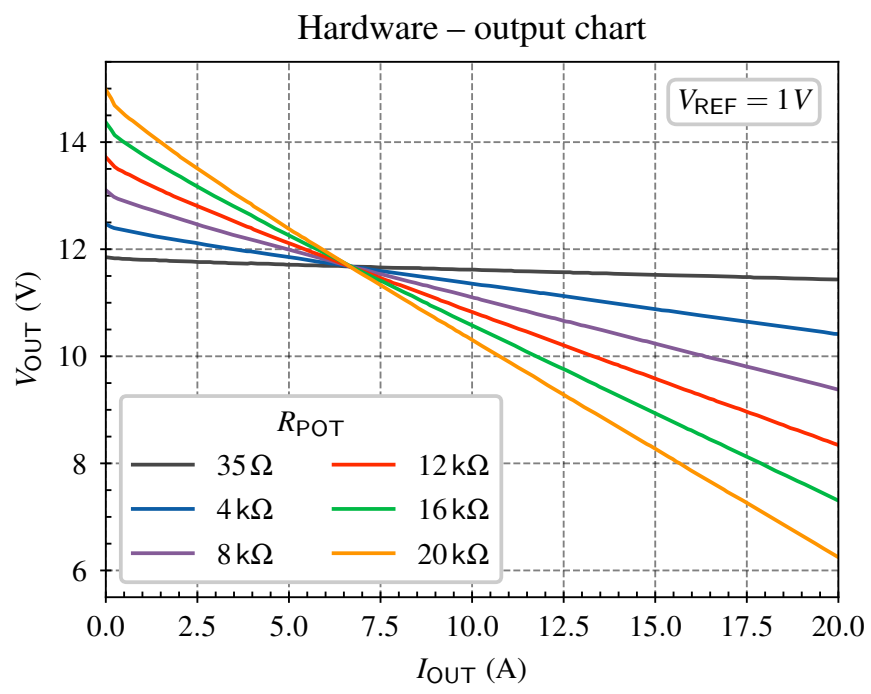

Fig. 9. Measurement results. The slight deviation from the simulation results shown in Fig. 7 is caused by a deviation of $V_{\text {REF }}$.

\section{Comparison}

To avoid artifacts in the derivation of the output characteristic field due to the quantization, the droop resistance is not displayed for the measurement on the hardware. Nevertheless, to compare the simulation with the hardware results, the droop resistance $R_{\mathrm{D}}$ was determined by performing linear regression in the range of $5 \mathrm{~A}$ to $20 \mathrm{~A}$ on the output characteristic fields (Figs. 7 and 9). Tab. 1 compares the values of the droop resistance $R_{\mathrm{D}}$ for the different resistor values of $R_{\mathrm{POT}}$.

Tab. 1. Comparision between simulation and test setup

\begin{tabular}{ccc}
\hline $\begin{array}{c}\text { Value of } R_{\mathrm{POT}} \\
(\Omega)\end{array}$ & $\begin{array}{c}R_{\mathrm{D}} \text { in Simulation } \\
(\mathrm{m} \Omega)\end{array}$ & $\begin{array}{c}R_{\mathrm{D}} \text { in Test setup } \\
(\mathrm{m} \Omega)\end{array}$ \\
\hline 35 & 18.27 & 18.44 \\
$2 \mathrm{k}$ & 56.08 & 56.39 \\
$4 \mathrm{k}$ & 94.56 & 95.27 \\
$6 \mathrm{k}$ & 133.0 & 134.2 \\
$8 \mathrm{k}$ & 171.5 & 173.2 \\
$10 \mathrm{k}$ & 210.0 & 212.2 \\
$12 \mathrm{k}$ & 248.5 & 250.5 \\
$14 \mathrm{k}$ & 287.0 & 290.3 \\
$16 \mathrm{k}$ & 325.4 & 329.2 \\
$18 \mathrm{k}$ & 363.9 & 368.1 \\
$20 \mathrm{k}$ & 402.4 & 407.7 \\
\hline
\end{tabular}

The comparison in Tab. 1 between the column for the simulation and the hardware construction shows that the droop resistance values differ only slightly. The deviations of up to $1.3 \%$ can be attributed to the tolerances of the components used. Furthermore, the systematic error between simulation and hardware is identifiable, which can be compensated by the microcontroller.

\section{CONCLUSION}

We have demonstrated that the circuit of a proportional amplifier is suitable to generate a digitally adjustable virtual internal resistance for the DC-DC converter used here. This was proven by a simulation and measurements on a real setup. As shown in Fig. 8, the resulting droop resistance is linear



Fig. 10. Underlaid evaluation board [17] with the attached $\mu \mathrm{C}$ - addon board. (1) \& (2) phase-shifted power stages, (3) circuit breaker, (4) eduacation board LM5170, (5) $\mathrm{\mu C}$ - add-on board. 
in a wide range, but not for small currents. Whether this behavior is undesirable must be clarified depending on the application. Due to the use of a voltage divider, no value of the droop resistor close to $0 \Omega$ can be set. Using a voltage follower operational amplifier circuit, this characteristic and the dependence of $R_{\mathrm{POT}}$ on $G_{\mathrm{VD}}$ (see equation 5) can be solved. Moreover, the settings for the bidirectional DC-DC converter can be conveniently made by the microcontroller and its peripherals (GPIO, $\left.\mathrm{I}^{2} \mathrm{C}, \mathrm{DAC}\right)$. However, a failure of the microcontroller leads to a change of the reference voltage $V_{\mathrm{REF}}$, since this is provided by the DAC. Consequently, this changes the output voltage $V_{\text {OUT }}$ of the DC-DC converter.

In conclusion, it can be confirmed that the circuit presented here fulfills the function of an analog voltage control with a digitally adjustable droop resistor very well. Thus, the combination of a DC-DC converter with a $\mu \mathrm{C}$ - add-on board can be used for the application as a node in a BMS. Also, the microcontroller together with a CAN communication can be used to implement a higher-level digital control. As a result, this system is suitable for load sharing of parallel connected components. Additionally, it is advantageous that the last settings of the parameters are retained in the event of a communication failure. So the droop-controlled bidirectional DC-DC converter with CAN bus communication is an important functioning component for the implementation of a BMS.

In the following work, the parallel connection of several DCDC converters with adjustable droop resistance is investigated. The aim is to still control a decentralized BMS in simulation [24] and in hardware tests. Furthermore, a compact all-in-one printed circuit board is currently designed, which will contain the power electronics of the DC-DC converter, as well as the analog voltage regulation with a digital steered droop parameter. In addition, the chosen microcontroller will also be able to communicate via CAN FD [25], which allows the control of multiple nodes by a single message. Moreover, the fail-safe functionality is improved so that the DC-DC converter with its communication-less control can be used for power sharing in a decentralized battery system.

\section{ACKNOWLEDGMENT}

The authors thank Michael Farmbauer of the Ostbayerische Technische Hochschule Regensburg for helpful discussions and support in the PCB design.

\section{REFERENCES}

[1] A. Chub, D. Vinnikov, R. Kosenko, E. Liivik, and I. Galkin, "Bidirectional DC-DC Converter for Modular Residential Battery Energy Storage Systems," in IEEE Transactions on Industrial Electronics, vol. 67, no. 3, pp. 1944-1955, March 2020, doi: 10.1109/TIE.2019.2902828

[2] N. Kondrath, "Bidirectional DC-DC converter topologies and control strategies for interfacing energy storage systems in microgrids: An overview," 2017 IEEE International Conference on Smart Energy Grid Engineering (SEGE), 2017, pp. 341-345, doi 10.1109/SEGE.2017.8052822.

[3] K. Tytelmaier, O. Husev, O. Veligorskyi, and R. Yershov, "A review of non-isolated bidirectional dc-dc converters for energy storage systems," 2016 II International Young Scientists Forum on Applied Physics and Engineering (YSF), 2016, pp. 22-28, doi: 10.1109/YSF.2016.7753752.

[4] Y. Gu, X. Xiang, W. Li, and X. He, "Mode-Adaptive Decentralized Control for Renewable DC Microgrid With Enhanced Reliability and Flexibility," in IEEE Transactions on Power Electronics, vol. 29, no. 9, pp. 5072-5080, Sept. 2014, doi: 10.1109/TPEL.2013.2294204.
[5] K. D. Hoang and H. Lee, "Accurate Power Sharing With Balanced Battery State of Charge in Distributed DC Microgrid," in IEEE Transactions on Industrial Electronics, vol. 66, no. 3, pp. 1883-1893, March 2019, doi: 10.1109/TIE.2018.2838107.

[6] K. R. Bharath, C. Harsha, and P. Kanakasabapathy, "Control of Bidirectional DC-DC Converter in Renewable based DC Microgrid with Improved Voltage Stability," Int. Journal of Renewable Energy Research, vol. 8, no. 2, pp. 871-877, June 2018.

[7] N. Zhi, K. Ding, L. Du, and H. Zhang, "An SOC-Based Virtual DC Machine Control for Distributed Storage Systems in DC Microgrids," in IEEE Transactions on Energy Conversion, vol. 35, no. 3, pp. 1411-1420, Sept. 2020, doi: 10.1109/TEC.2020.2975033.

[8] S. Anand, B. G. Fernandes, and J. Guerrero, "Distributed Control to Ensure Proportional Load Sharing and Improve Voltage Regulation in LowVoltage DC Microgrids," in IEEE Transactions on Power Electronics, vol. 28, no. 4, pp. 1900-1913, April 2013, doi: 10.1109/TPEL.2012.2215055

[9] P. Prabhakaran, Y. Goyal, and V. Agarwal, "Novel Nonlinear Droop Control Techniques to Overcome the Load Sharing and Voltage Regulation Issues in DC Microgrid," in IEEE Transactions on Power Electronics, vol. 33, no. 5, pp. 4477-4487, May 2018, doi: 10.1109/TPEL.2017.2723045.

10] P. Huang, P. Liu, W. Xiao, and M. S. El Moursi, "A Novel Droop-Based Average Voltage Sharing Control Strategy for DC Microgrids," in IEEE Transactions on Smart Grid, vol. 6, no. 3, pp. 1096-1106, May 2015 , doi: $10.1109 /$ TSG.2014.2357179.

[11] X. Lu, K. Sun, J. M. Guerrero, J. C. Vasquez, and L. Huang, "State-ofCharge Balance Using Adaptive Droop Control for Distributed Energy Storage Systems in DC Microgrid Applications," in IEEE Transactions on Industrial Electronics, vol. 61, no. 6, pp. 2804-2815, June 2014, doi: 10.1109/TIE.2013.2279374.

[12] A. Reindl, H. Meier, and M. Niemetz, "Scalable, Decentralized Battery Management System Based on Self-organizing Nodes," Architecture of Computing Systems, Springer, 2020, doi: 10.1007/978-3-030-527945_13.

[13] G. Aulagnier, K. Abouda, E. Rolland, M. Cousineau, and T. Meynard, "Benefits of multiphase Buck converters in reducing EME (Electromagnetic Emissions) Analysis and application to on-chip converters for automotive applications," 2015 IEEE International Symposium on Electromagnetic Compatibility (EMC), 2015, pp. 102-107, doi: 10.1109/ISEMC.2015.7256140.

[14] R. M. Schupbach and J. C. Balda, "Comparing DC-DC converters for power management in hybrid electric vehicles," IEEE International Electric Machines and Drives Conference, 2003, pp. 1369-1374 vol.3, doi: 10.1109/IEMDC.2003.1210630.

[15] E. Ribeiro, A. J. M. Cardoso, and C. Boccaletti, "Modular hybrid storage system for renewable energy standalone power supplies," IECON 2013 - 39th Annual Conference of the IEEE Industrial Electronics Society, 2013, pp. 1749-1754, doi: 10.1109/IECON.2013.6699396.

[16] Texas Instruments, "Multiphase bidirectional current controller," LM5170 datasheet, Dec. 2019 [Revised June 2020]

[17] Texas Instruments, "LM5170 48V-12V Bidirectional Converter Evaluation Module," LM5170EVM-BIDIR datasheet, Nov. 2016 [Revised Dec. 2016].

[18] STMicroelectronics, "Ultra-low-power with FPU Arm Cortex-M4 MCU $80 \mathrm{MHz}$ with 256 Kbytes of Flash memory, LCD, USB," STM32L433RC datasheet, Feb. 2016 [Revised May. 2018].

[19] I. Batarseh, K. Siri, and H. Lee, "Investigation of the output droop characteristics of parallel-connnected DC-DC converters," Proceedings of 1994 Power Electronics Specialist Conference - PESC'94, 1994, pp. 1342-1351 vol.2, doi: 10.1109/PESC.1994.373859.

[20] H. Wang, M. Han, J. M. Guerrero, J. C. Vasquez, and R. Han, ”The influence of internal current loop on transient response performance of I-V droop controlled paralleled DC-DC converters," $43^{\text {rd }}$ Conference of the IEEE Industrial Electronics Society, 2017, pp. 4982-4987, doi 10.1109/IECON.2017.8216860.

[21] Analog Devices, "1024-/256-Position, 1\% Resistor Tolerance Error $\mathrm{I}^{2} \mathrm{C}$ Interface and 50-TP Memory Digital Rheostat," AD5272 datasheet, Oct. 2009 [Revised Mar. 2013].

[22] M. Engelhardt, Linear Technology and Analog Devices, "LTspice XVII" 2021.

[23] Elektro-Automatik, "EL 3000 B Electronic DC Load," EA-EL 3080-60 B datasheet, Nov. 2020 [Revision: 02].

[24] A. Reindl, H. Meier, and M. Niemetz, "Software Framework for the Simulation of a Decentralized Battery Management System Consisting of Intelligent Battery Cells,” 2019 IEEE Student Conference on Research and Development (SCOReD), 2019, pp. 75-80, doi: 10.1109/SCORED.2019.8896284

[25] A. Reindl, D. Wetzel, N. Balbierer, H. Meier, M. Niemetz, and S. Park, "Comparative Analysis of CAN, CAN FD and Ethernet for Networked Control Systems," Embedded World Conference DIGITAL, Mar. 2021. 